\title{
QUASIMODULAR FORMS AND VECTOR BUNDLES
}

\author{
MIN HO LEE
}

(Received 28 November 2008)

\begin{abstract}
Modular forms for a discrete subgroup $\Gamma$ of $\operatorname{SL}(2, \mathbb{R})$ can be identified with holomorphic sections of line bundles over the modular curve $U$ corresponding to $\Gamma$, and quasimodular forms generalize modular forms. We construct vector bundles over $U$ whose sections can be identified with quasimodular forms for $\Gamma$.
\end{abstract}

2000 Mathematics subject classification: primary $11 \mathrm{~F} 11$.

Keywords and phrases: quasimodular forms, modular forms, vector bundles.

\section{Introduction}

Modular forms for a discrete subgroup $\Gamma$ of $S L(2, \mathbb{R})$ are closely linked to the geometry of the quotient $\Gamma \backslash \mathcal{H}$ of the Poincaré upper half plane $\mathcal{H}$ by the linear fractional action of $\Gamma$. One such link is given by the interpretation of modular forms as holomorphic sections of line bundles over $\Gamma \backslash \mathcal{H}$. The goal of this paper is to extend such interpretation to the case of quasimodular forms.

Quasimodular forms generalize classical modular forms and were introduced by Kaneko and Zagier in [3]. Since then, they have been studied actively not only in number theory but also in other branches of pure and applied mathematics (see, for example, $[2,4,5])$. One of the useful properties of quasimodular forms is that, unlike modular forms, derivatives of quasimodular forms are also quasimodular forms. If $f$ is a quasimodular form for $\Gamma$ of weight $w$ and depth at most $m \geq 0$, then there are holomorphic functions $f_{0}, f_{1}, \ldots, f_{m}$ on $\mathcal{H}$ satisfying

$$
\frac{1}{(c z+d)^{w}} f\left(\frac{a z+b}{c z+d}\right)=f_{0}(z)+f_{1}(z)\left(\frac{c}{c z+d}\right)+\cdots+f_{m}(z)\left(\frac{c}{c z+d}\right)^{m}
$$

for all $z \in \mathcal{H}$ and $\left(\begin{array}{ll}a & b \\ c & d\end{array}\right) \in \Gamma$. Then it can be shown that the associated polynomial

$$
F(z, X)=\sum_{r=0}^{m} f_{r}(z) X^{r}
$$

This research was supported in part by a PDA award from the University of Northern Iowa.

(C) 2009 Australian Mathematical Publishing Association Inc. 0004-9727/2009 \$16.00 
known as a quasimodular polynomial, is invariant under a certain right action of $\Gamma$. In fact, the above correspondence determines an isomorphism between the space of quasimodular forms and that of quasimodular polynomials.

In this paper we use the above-mentioned right action of $\Gamma$ on the space of quasimodular polynomials to construct vector bundles over $\Gamma \backslash \mathcal{H}$ whose sections can be identified with quasimodular polynomials and therefore with quasimodular forms.

\section{Quasimodular forms}

In this section we describe quasimodular forms for a discrete subgroup of $S L(2, \mathbb{R})$. We also discuss some basic properties of quasimodular polynomials, which can be identified with quasimodular forms.

Let $\mathcal{H}$ be the Poincaré upper half plane on which $\operatorname{SL}(2, \mathbb{R})$ acts as usual by linear fractional transformation. Thus, if $\gamma=\left(\begin{array}{ll}a & b \\ c & d\end{array}\right) \in S L(2, \mathbb{R})$ and $z \in \mathcal{H}$, we may write

$$
\gamma z=\frac{a z+b}{c z+d} \in \mathcal{H} \text {. }
$$

For the same $\gamma$ and $z$ we set

$$
\mathfrak{J}(\gamma, z)=c z+d, \quad \mathfrak{K}(\gamma, z)=\frac{c}{c z+d},
$$

so that we obtain the maps $\mathfrak{J}, \mathfrak{K}: S L(2, \mathbb{R}) \times \mathcal{H} \rightarrow \mathbb{C}$. The map $\mathfrak{J}$ is a well-known automorphy factor satisfying the cocycle condition

$$
\mathfrak{J}\left(\gamma \gamma^{\prime}, z\right)=\mathfrak{J}\left(\gamma, \gamma^{\prime} z\right) \mathfrak{J}\left(\gamma^{\prime}, z\right)
$$

for all $z \in \mathcal{H}$ and $\gamma \gamma^{\prime} \in S L(2, \mathbb{R})$. The other function $\mathfrak{K}$, on the other hand, satisfies

$$
\mathfrak{K}\left(\gamma \gamma^{\prime}, z\right)=\mathfrak{K}\left(\gamma^{\prime}, z\right)+\mathfrak{J}\left(\gamma^{\prime}, z\right)^{-2} \mathfrak{K}\left(\gamma, \gamma^{\prime} z\right) .
$$

Let $\mathcal{F}$ be the ring of holomorphic functions on $\mathcal{H}$, and denote by $\mathcal{F}_{m}[X]$ with $m \geq 0$ the complex algebra of polynomials in $X$ over $\mathcal{F}$ of degree at most $m$. Given elements $f \in \mathcal{F}, F(z, X) \in \mathcal{F}_{m}[X], \lambda \in \mathbb{Z}$, and $\gamma \in S L(2, \mathbb{R})$, we set

$$
\begin{gathered}
\left(\left.f\right|_{\lambda} \gamma\right)(z)=\mathfrak{J}(\gamma, z)^{-\lambda} f(z) \\
\left(F \|_{\lambda} \gamma\right)(z, X)=\mathfrak{J}(\gamma, z)^{-\lambda} F\left(\gamma z, \mathfrak{J}(\gamma, z)^{2}(X-\mathfrak{K}(\gamma, z))\right)
\end{gathered}
$$

for all $z \in \mathcal{H}$. If $\gamma^{\prime}$ is another element of $\operatorname{SL}(2, \mathbb{R})$, using (2.2) and (2.3), it can be shown that

$$
\begin{aligned}
\left.f\right|_{\lambda}\left(\gamma \gamma^{\prime}\right) & =\left.\left(\left.f\right|_{\lambda} \gamma\right)\right|_{\lambda} \gamma^{\prime}, \\
\left(\left(F \|_{\lambda} \gamma\right) \|_{\lambda} \gamma^{\prime}\right)(z, X) & =\left(F \|_{\lambda}\left(\gamma \gamma^{\prime}\right)\right)(z, X) .
\end{aligned}
$$

Thus the operations $\left.\right|_{\lambda}$ and $\|_{\lambda}$ determine right actions of $S L(2, \mathbb{R})$ on $\mathcal{F}$ and $\mathcal{F}_{m}[X]$, respectively.

We now consider a discrete subgroup $\Gamma$ of $S L(2, \mathbb{R})$ and modify the usual definition of modular and quasimodular forms for $\Gamma$ by suppressing the cusp conditions. 
DEFINITION 2.1.

(i) Given an integer $\mu$, an element $f \in \mathcal{F}$ is a modular form for $\Gamma$ of weight $\mu$ if it satisfies

$$
\left.f\right|_{\mu} \gamma=f
$$

for all $\gamma \in \Gamma$, where $\left.\right|_{\mu}$ is the operation in (2.4). We denote by $M_{\mu}(\Gamma)$ the space of modular forms for $\Gamma$ of weight $\mu$.

(ii) Given integers $\xi$ and $m$ with $m \geq 0$, an element $f \in \mathcal{F}$ is a quasimodular form for $\Gamma$ of weight $\xi$ and depth at most $m$ if there are functions $f_{0}, \ldots, f_{m} \in \mathcal{F}$ such that

$$
(f \mid \xi \gamma)(z)=\sum_{r=0}^{m} f_{r}(z) \mathfrak{K}(\gamma, z)^{r}
$$

for all $z \in \mathcal{H}$ and $\gamma \in \Gamma$, where $\mathfrak{K}(\gamma, z)$ is as in (2.3). We denote by $Q M_{\xi}^{m}(\Gamma)$ the space of quasimodular forms for $\Gamma$ of weight $\xi$ and depth at most $m$.

If $f \in Q M_{\xi}^{m}(\Gamma)$ is a quasimodular form satisfying (2.7), by using the identity element for $\gamma$, we obtain

$$
f(z)=f_{0}(z)
$$

for all $z \in \mathcal{H}$. On the other hand, for fixed $z \in \mathcal{H}$, by considering the right-hand side of (2.7) as a polynomial in $\mathfrak{K}(\gamma, z)$ and using the fact that the same equation is valid for all elements $\Gamma$ of $\Gamma$, we see that the given quasimodular form $f \in Q M_{\xi}^{m}(\Gamma)$ determines the coefficients $f_{0}, \ldots, f_{m}$ uniquely. We also see easily that

$$
Q M_{\xi}^{0}(\Gamma)=M_{\xi}(\Gamma)
$$

for each $\xi \in \mathbb{Z}$.

Given a quasimodular form $f \in Q M_{\xi}^{m}(\Gamma)$ satisfying (2.7), we define the corresponding polynomial $\left(\mathcal{Q}_{\xi}^{m} f\right)(z, X) \in \mathcal{\mathcal { F }}_{m}[X]$ by

$$
\left(\mathcal{Q}_{\xi}^{m} f\right)(z, X)=\sum_{r=0}^{m} f_{r}(z) X^{r}
$$

for $z \in \mathcal{H}$, so that we obtain the complex linear map

$$
\mathcal{Q}_{\xi}^{m}: Q M_{\xi}^{m}(\Gamma) \rightarrow \mathcal{F}_{m}[X]
$$

for each pair of nonnegative integers $\xi$ and $m$.

DEFINITION 2.2. A quasimodular polynomial for $\Gamma$ of weight $\xi$ and degree at most $m$ is an element of $\mathcal{F}_{m}[X]$ that is $\Gamma$-invariant with respect to the right $\Gamma$-action in (2.5). We denote by

$$
Q P_{\xi}^{m}(\Gamma)=\left\{F(z, X) \in \mathcal{F}_{m}[X] \mid F \|_{\xi} \gamma=F \text { for all } \gamma \in \Gamma\right\}
$$

the space of all quasimodular polynomials for $\Gamma$ weight $\xi$ and degree at most $m$. 
LEMMA 2.3.

(i) If $f \in \mathcal{F}$ is a quasimodular form belonging to $Q M_{\xi}^{m}(\Gamma)$, then

$$
\left(\mathcal{Q}_{\xi}^{m} f\right)(z, X) \in Q P_{\xi}^{m}(\Gamma) .
$$

(ii) Let $F(z, X)$ be a quasimodular polynomial of the form

$$
F(z, X)=\sum_{r=0}^{m} f_{r}(z) X^{r}
$$

belonging to $Q P_{\xi}^{m}(\Gamma)$. Then $f_{0}$ is a quasimodular form belonging to $Q M_{\xi}^{m}(\Gamma)$ such that the condition (2.7) is satisfied for $f=f_{0}$. Furthermore, for each $r \in\{0,1, \ldots, m\}$ the coefficient $f_{r}$ satisfies

$$
\left(\left.f_{r}\right|_{\xi-2 r} \gamma\right)(z)=\sum_{\ell=r}^{m}\left(\begin{array}{l}
\ell \\
r
\end{array}\right) f_{\ell}(z) \mathfrak{K}(\gamma, z)^{\ell-r}=\sum_{\ell=0}^{m-r}\left(\begin{array}{c}
\ell+r \\
r
\end{array}\right) f_{\ell+r}(z) \mathfrak{K}(\gamma, z)^{\ell}
$$

for all $z \in \mathcal{H}$ and $\gamma \in \Gamma$.

PROOF. These results can be proved by using the definition of the operation $\|_{\xi}$ in (2.5) and the relations in (2.2) and (2.3) (see, for example, [1]).

By Lemma 2.3 the map $\mathcal{Q}_{\xi}^{m}$ given by (2.8) induces an isomorphism

$$
\mathcal{Q}_{\xi}^{m}: Q P_{\xi}^{m}(\Gamma) \rightarrow Q M_{\xi}^{m}(\Gamma) .
$$

Furthermore, if $\mathcal{Q}_{\xi}^{m} f$ with $f \in Q P_{\xi}^{m}(\Gamma)$ is as in (2.8), then

$$
f_{0}=f \in Q M_{\xi}^{m}(\Gamma)
$$

hence, the inverse of the isomorphism $\mathcal{Q}_{\xi}^{m}$ is the map

$$
\mathcal{P}_{0}: Q P_{\xi}^{m}(\Gamma) \rightarrow Q M_{\xi}^{m}(\Gamma)
$$

sending a quasimodular polynomial $F(z, X) \in Q P_{\xi}^{m}(\Gamma)$ to its constant term

$$
\left(\mathcal{P}_{0} F\right)(z)=F(z, 0)
$$

for all $z \in \mathcal{H}$.

\section{Vector bundles}

Let $\Gamma$ be a discrete subgroup of $S L(2, \mathbb{R})$ as in Section 2 . In this section we construct vector bundles over the quotient space $\Gamma \backslash \mathcal{H}$ whose sections may be identified with quasimodular polynomials and therefore quasimodular forms for $\Gamma$. 
Given integers $\lambda, k$ and $r$ with $0 \leq k \leq r \leq m$, we consider a map

$$
\Xi_{r}^{\lambda, k}: S L(2, \mathbb{R}) \times \mathcal{H} \rightarrow \mathbb{C}
$$

defined by

$$
\Xi_{r}^{\lambda, k}(\gamma, z)=\left(\begin{array}{l}
k \\
r
\end{array}\right) \mathfrak{J}(\gamma, z)^{\lambda-2 r} \mathfrak{K}(\gamma, z)^{k-r}
$$

for $\gamma \in S L(2, \mathbb{R})$ and $z \in \mathcal{H}$.

LEMMA 3.1. The map $\Xi_{r}^{\lambda, k}$ given by (3.1) satisfies

$$
\Xi_{r}^{\lambda, k}\left(\gamma_{1} \gamma, z\right)=\sum_{\ell=r}^{k} \Xi_{r}^{\lambda, \ell}\left(\gamma_{1}, \gamma z\right) \Xi_{\ell}^{\lambda, k}(\gamma, z)
$$

for all $\gamma_{1}, \gamma \in S L(2, \mathbb{R})$ and $z \in \mathcal{H}$.

PROOF. If $\gamma_{1}, \gamma \in S L(2, \mathbb{R})$ and $z \in \mathcal{H}$, from (3.1) we obtain

$$
\Xi_{r}^{\lambda, k}\left(\gamma_{1} \gamma, z\right)=\left(\begin{array}{l}
k \\
r
\end{array}\right) \mathfrak{J}\left(\gamma_{1} \gamma, z\right)^{\lambda-2 r} \mathfrak{K}\left(\gamma_{1} \gamma, z\right)^{k-r} .
$$

However, using (2.2) and (2.3),

$$
\begin{aligned}
\mathfrak{J}\left(\gamma_{1} \gamma, z\right)^{\lambda-2 r} & =\mathfrak{J}\left(\gamma_{1}, \gamma z\right)^{\lambda-2 r} \mathfrak{J}(\gamma, z)^{\lambda-2 r}, \\
\mathfrak{K}\left(\gamma_{1} \gamma, z\right)^{k-r} & =\left(\mathfrak{K}(\gamma, z)+\mathfrak{J}(\gamma, z)^{-2} \mathfrak{K}\left(\gamma_{1}, \gamma z\right)\right)^{k-r} \\
& =\sum_{j=0}^{k-r}\left(\begin{array}{c}
k-r \\
j
\end{array}\right) \mathfrak{J}(\gamma, z)^{-2 j} \mathfrak{K}\left(\gamma_{1}, \gamma z\right)^{j} \mathfrak{K}(\gamma, z)^{k-r-j} \\
& =\sum_{\ell=r}^{k}\left(\begin{array}{c}
k-r \\
\ell-r
\end{array}\right) \mathfrak{J}(\gamma, z)^{-2 \ell+2 r} \mathfrak{K}\left(\gamma_{1}, \gamma z\right)^{\ell-r} \mathfrak{K}(\gamma, z)^{k-\ell} .
\end{aligned}
$$

Hence, we see that

$$
\begin{aligned}
\Xi_{r}^{\lambda, k}\left(\gamma_{1} \gamma, z\right)=\sum_{\ell=r}^{k} & \left(\begin{array}{l}
k \\
r
\end{array}\right)\left(\begin{array}{l}
k-r \\
\ell-r
\end{array}\right) \mathfrak{J}\left(\gamma_{1}, \gamma z\right)^{\lambda-2 r} \mathfrak{J}(\gamma, z)^{\lambda-2 \ell} \\
& \times \mathfrak{K}\left(\gamma_{1}, \gamma z\right)^{\ell-r} \mathfrak{K}(\gamma, z)^{k-\ell}
\end{aligned}
$$

On the other hand,

$$
\begin{aligned}
\Xi_{r}^{\lambda, \ell}\left(\gamma_{1}, \gamma z\right) \Xi_{\ell}^{\lambda, k}(\gamma, z)=\left(\begin{array}{l}
\ell \\
r
\end{array}\right) & \mathfrak{J}\left(\gamma_{1}, \gamma z\right)^{\lambda-2 r} \mathfrak{K}\left(\gamma_{1}, \gamma z\right)^{\ell-r} \\
& \times\left(\begin{array}{l}
k \\
\ell
\end{array}\right) \mathfrak{J}(\gamma, z)^{\lambda-2 \ell} \mathfrak{K}(\gamma, z)^{k-\ell} \\
= & \left(\begin{array}{l}
k \\
\ell
\end{array}\right) \\
& \times\left(\begin{array}{l}
\ell \\
r
\end{array}\right) \mathfrak{J}\left(\gamma_{1}, \gamma z\right)^{\lambda-2 r} \mathfrak{J}(\gamma, z)^{\lambda-2 \ell} \\
& \times \mathfrak{K}\left(\gamma_{1}, \gamma z\right)^{\ell-r} \mathfrak{K}(\gamma, z)^{k-\ell} .
\end{aligned}
$$


From (3.3), (3.4) and the relation

$$
\left(\begin{array}{l}
k \\
r
\end{array}\right)\left(\begin{array}{l}
k-r \\
\ell-r
\end{array}\right)=\frac{k !}{r !(\ell-r) !(k-\ell) !}=\left(\begin{array}{l}
k \\
\ell
\end{array}\right)\left(\begin{array}{l}
\ell \\
r
\end{array}\right),
$$

formula (3.2) follows.

We fix a nonnegative integer $m$, and denote by $\mathbb{C}_{m}[X]$ the ring of polynomials in $X$ over $\mathbb{C}$ of degree at most $m$. Given a polynomial of the form

$$
F(X)=\sum_{r=0}^{m} c_{r} X^{r} \in \mathbb{C}_{m}[X]
$$

with $c_{0}, \ldots, c_{m} \in \mathbb{C}$ and an integer $\lambda$, we now set

$$
\gamma \odot_{\lambda}^{m}(z, F(X))=\left(\gamma z, \sum_{r=0}^{m} \sum_{k=r}^{m} c_{k} \Xi_{r}^{\lambda, k}(\gamma, z) X^{r}\right)
$$

for all $\gamma \in S L(2, \mathbb{R})$ and $z \in \mathcal{H}$.

Proposition 3.2. Equation (3.6) determines a left action of $\operatorname{SL}(2, \mathbb{R})$ on the Cartesian product $\mathcal{H} \times \mathbb{C}_{m}[X]$.

PROOF. Given elements $\gamma, \gamma_{1} \in S L(2, \mathbb{R}), z \in \mathcal{H}$ and a polynomial $F(X) \in \mathbb{C}_{m}[X]$ as in (3.5), using (3.6), we obtain

$$
\begin{aligned}
\gamma_{1} \odot_{\lambda}^{m}\left(\gamma \odot_{\lambda}^{m}(z, F(X))\right) & =\left(\gamma_{1} \gamma z, \sum_{r=0}^{m} \sum_{\ell=r}^{m} \sum_{k=\ell}^{m} c_{k} \Xi_{\ell}^{\lambda, k}(\gamma, z) \Xi_{r}^{\lambda, \ell}(\gamma, z) X^{r}\right) \\
& =\left(\gamma_{1} \gamma z, \sum_{r=0}^{m} \sum_{k=r}^{m} \sum_{\ell=r}^{k} c_{k} \Xi_{\ell}^{\lambda, k}(\gamma, z) \Xi_{r}^{\lambda, \ell}(\gamma, z) X^{r}\right) .
\end{aligned}
$$

On the other hand,

$$
\left(\gamma_{1} \gamma\right) \odot_{\lambda}^{m}(z, F(X))=\left(\gamma_{1} \gamma z, \sum_{r=0}^{m} \sum_{k=r}^{m} c_{k} \Xi_{\ell}^{\lambda, k}\left(\gamma_{1} \gamma, z\right) X^{r}\right) .
$$

Combining (3.7) and (3.8) with (3.2), we obtain

$$
\gamma_{1} \odot_{\lambda}^{m}\left(\gamma \odot_{\lambda}^{m}(z, F(X))\right)=\left(\gamma_{1} \gamma\right) \odot_{\lambda}^{m}(z, F(X))
$$

hence, the proposition follows.

Let $\Gamma$ be a discrete subgroup of $\operatorname{SL}(2, \mathbb{R})$, and denote the quotient of the space $\mathcal{H} \times \mathbb{C}_{m}[X]$ by $\Gamma$ with respect to the action shown in Proposition 3.2 by

$$
\mathcal{V}_{\lambda}^{m}=\Gamma \backslash \mathcal{H} \times \mathbb{C}_{m}[X] .
$$


If we denote the modular curve associated with $\Gamma$ by $U=\Gamma \backslash \mathcal{H}$, then the natural projection map $\mathcal{H} \times \mathbb{C}_{m}[X] \rightarrow \mathcal{H}$ induces a surjective map $\varpi: \mathcal{V}_{\lambda}^{m} \rightarrow U$ such that $\varpi^{-1}(x)$ is isomorphic to $\mathbb{C}_{m}[X]$ for each $x \in U$. Thus $\mathcal{V}_{\lambda}^{m}$ has the structure of a complex vector bundle over $U$ whose fiber is the $(m+1)$-dimensional complex vector space $\mathbb{C}_{m}[X]$ of polynomials in $X$. We denote by $\boldsymbol{\Gamma}_{0}\left(U, \mathcal{V}_{\lambda}^{m}\right)$ the space of all holomorphic sections of $\mathcal{V}_{\lambda}^{m}$ over $U$.

THEOREM 3.3. The space $\Gamma_{0}\left(U, \mathcal{V}_{\lambda}^{m}\right)$ of holomorphic sections of $\mathcal{V}_{\lambda}^{m}$ over $U=\Gamma \backslash \mathcal{H}$ is canonically isomorphic to the space $Q P_{\lambda}^{m}(\Gamma)$ of all quasimodular polynomials for $\Gamma$ of weight $\lambda$ and depth at most $m$.

Proof. Let $\sigma: U \rightarrow \mathcal{V}_{\lambda}^{m}$ be a holomorphic section of $\mathcal{V}_{\lambda}^{m}$ over $U=\Gamma \backslash \mathcal{H}$, and denote by $q: \mathcal{H} \rightarrow U$ the natural projection map. Given $z \in \mathcal{H}$, then

$$
\sigma(q(z))=\left[\left(z, \sum_{r=0}^{m} c_{r, z} X^{r}\right)\right] \in \Gamma \backslash \mathcal{H} \times \mathbb{C}_{m}[X]
$$

for some $c_{0, z}, \ldots, c_{m, z} \in \mathbb{C}$, where $[(\cdot)]$ denotes the $\Gamma$-orbit of the element (.) of $\mathcal{H} \times \mathbb{C}_{m}[X]$. We define the $\mathbb{C}$-valued functions $f_{0}^{\sigma}, \ldots, f_{m}^{\sigma}$ on $\mathcal{H}$ by

$$
f_{r}^{\sigma}(z)=c_{r, z}
$$

for all $z \in \mathcal{H}$ and $0 \leq r \leq m$. Given $\gamma \in \Gamma$, since for each $z \in \mathcal{H}$ the $\Gamma$-orbits of $z$ and $\gamma z$ are the same, then

$$
\sigma(q(z))=\sigma(q(\gamma z))=\left[\left(\gamma z, \sum_{r=0}^{m} c_{r, \gamma z} X^{r}\right)\right] .
$$

On the other hand, since

$$
\left[\gamma \odot_{\lambda}^{m}(z, f(X))\right]=[(z, f(X))]
$$

for each $(z, f(X)) \in \mathcal{H} \times \mathbb{C}_{m}[X]$, using (3.6) leads to

$$
\begin{aligned}
\sigma(q(z)) & =\left[\gamma \odot_{\lambda}^{m}\left(z, \sum_{r=0}^{m} c_{r, z} X^{r}\right)\right] \\
& =\left[\left(\gamma z, \sum_{r=0}^{m} \sum_{k=r}^{m} c_{k, z} \Xi_{r}^{\lambda, k}(\gamma, z) X^{r}\right)\right] .
\end{aligned}
$$

Comparing this with (3.11) and using (3.1) and (3.10), we see that

$$
\begin{aligned}
f_{r}^{\sigma}(\gamma z)=c_{r, \gamma z} & =\sum_{k=r}^{m} c_{k, z} \Xi_{r}^{\lambda, k}(\gamma, z) \\
& =\sum_{k=r}^{m}\left(\begin{array}{l}
k \\
r
\end{array}\right) \mathfrak{J}(\gamma, z)^{\lambda-2 r} \mathfrak{K}(\gamma, z)^{k-r} f_{k}^{\sigma}(z) ;
\end{aligned}
$$


hence, we obtain

$$
\left(\left.f_{r}^{\sigma}\right|_{\lambda-2 r} \gamma\right)(z)=\sum_{k=r}^{m}\left(\begin{array}{l}
k \\
r
\end{array}\right) \mathfrak{K}(\gamma, z)^{k-r} f_{k}^{\sigma}(z)
$$

for $0 \leq r \leq m$. In particular,

$$
\left(\left.f_{0}^{\sigma}\right|_{\lambda-2 r} \gamma\right)(z)=\sum_{k=0}^{m} \mathfrak{K}(\gamma, z)^{k-r} f_{k}^{\sigma}(z),
$$

and therefore it follows that the polynomial

$$
F^{\sigma}(z, X)=\sum_{r=0}^{m} f_{r}^{\sigma}(z) X^{r}
$$

is a quasimodular polynomial belonging to $Q P_{\lambda}^{m}(\Gamma)$. On the other hand, we assume that $G(z, X)=\sum_{r=0}^{m} g_{r}(z) X^{r}$ is a quasimodular polynomial belonging to $Q P_{\lambda}^{m}(\Gamma)$. We define the map $\sigma_{G}: U \rightarrow \mathcal{V}_{\lambda}^{m}$ by

$$
\sigma_{G}(q(z))=\left[\left(z, \sum_{r=0}^{m} g_{r}(z) X^{r}\right)\right]
$$

for all $z \in \mathcal{H}$. Then for each $\gamma \in \Gamma$, using (2.9), (3.1) and (3.6),

$$
\begin{aligned}
\sigma_{G}(q(\gamma z)) & =\left[\left(\gamma z, \sum_{k=0}^{m} g_{k}(\gamma z) X^{k+\delta}\right)\right] \\
& =\left[\left(\gamma z, \sum_{k=0}^{m} \sum_{k=r}^{m}\left(\begin{array}{l}
k \\
r
\end{array}\right) \mathfrak{J}(\gamma, z)^{\lambda-2 r} \mathfrak{K}(\gamma, z)^{k-r} g_{k}(z)\right)\right] \\
& =\left[\left(\gamma z, \sum_{k=0}^{m} \sum_{k=r}^{m} \Xi_{r}^{\lambda, k}(\gamma, z) g_{k}(z)\right)\right] \\
& =\left[\gamma \odot_{\lambda}^{m}\left(z, \sum_{r=0}^{m} g_{r}(z) X^{r}\right)\right],
\end{aligned}
$$

and therefore $\sigma_{\Gamma}$ is well defined. Since clearly $\varpi \circ \sigma_{G}=1_{U}$, it follows that $\sigma_{G}$ is a holomorphic section of $\mathcal{V}_{\lambda}^{m}$ over $U$; hence, the proof of the theorem is complete.

REMARK 3.4. If $m=0$, then the bundle $\mathcal{V}_{\lambda}^{0}$ becomes a line bundle and we obtain the isomorphism

$$
\Gamma_{0}\left(U, \mathcal{V}_{\lambda}^{m}\right) \cong M_{\lambda}(\Gamma)
$$

for each $\lambda$, which provides the usual identification between modular forms and holomorphic sections of a line bundle. 


\section{Morphisms of vector bundles}

Given $m \geq 0$, there are natural linear maps carrying quasimodular forms of depth at most $m$ to those of depth at most $r \leq m$. In this section we construct morphisms of vector bundles over a modular curve corresponding to such linear maps.

Given a polynomial $F(z, X) \in \mathcal{F}_{m}[X]$ of the form

$$
F(z, X)=\sum_{r=0}^{m} f_{r}(z) X^{r}
$$

with $f_{0}, \ldots, f_{m} \in \mathcal{F}$, we set

$$
\left(\Delta_{p} F\right)(z, X)=\sum_{r=0}^{m-p}\left(\begin{array}{c}
r+p \\
p
\end{array}\right) f_{r+p}(z) X^{r}
$$

for each integer $p$ with $0 \leq p \leq m$, so that we obtain the complex linear map

$$
\Delta_{p}: \mathcal{F}_{m}[X] \rightarrow \mathcal{F}_{m-p}[X] .
$$

LEMMA 4.1. Given $\lambda \in \mathbb{Z}$, then

$$
\Delta_{p}\left(Q P_{p}^{m}(\Gamma)\right) \subset Q P_{\lambda-2 p}^{m-p}(\Gamma)
$$

for each $p \in\{0,1, \ldots, m\}$.

Proof. Let $F(z, X) \in Q P_{\lambda}^{m}(\Gamma)$ be a quasimodular polynomial of the form given by (4.1). Then by Lemma 2.3(ii) the coefficients of $F(z, X)$ satisfies

$$
\left(\left.f_{j}\right|_{\lambda-2 j} \gamma\right)(z)=\sum_{\ell=j}^{m}\left(\begin{array}{l}
\ell \\
j
\end{array}\right) f_{\ell}(z) \mathfrak{K}(\gamma, z)^{\ell-j}
$$

for all $z \in \mathcal{H}$ and $\gamma \in \Gamma$. From this and Lemma 2.3(i) we see that $f_{p}$ is a quasimodular form belonging to $Q F_{\lambda-2 p}^{m-p}(\Gamma)$, and therefore $\left(\Delta_{p} F\right)(z, X)$ is a quasimodular polynomial belonging to $Q P_{\lambda-2 p}^{m-p}(\Gamma)$.

Given $p \in\{0,1, \ldots, m\}$, we now define the map

$$
\widetilde{\Delta}_{p}: \mathcal{H} \times \mathbb{C}_{m}[X] \rightarrow \mathcal{H} \times \mathbb{C}_{m-p}[X]
$$

by

$$
\widetilde{\Delta}_{p}(z, f(X))=\left(z, \Delta_{p} f(X)\right)
$$

for all $f(X) \in \mathbb{C}_{m}[X]$, where $\Delta_{p}: \mathbb{C}_{m}[X] \rightarrow \mathbb{C}_{m-p}[X]$ is the map obtained from (4.3) by restriction. We consider the vector bundles

$$
\mathcal{V}_{\lambda}^{m}=\Gamma \backslash \mathcal{H} \times \mathbb{C}_{m}[X], \quad \mathcal{V}_{\lambda-2 p}^{m-p}=\Gamma \backslash \mathcal{H} \times \mathbb{C}_{m-p}[X],
$$

where the first bundle is as in (3.9) and the second quotient is with respect to the operation $\odot_{\lambda-2 p}^{m-p}$ in (3.6) of $\Gamma$ on $\mathcal{H} \times \mathbb{C}_{m-p}[X]$. 
THEOREM 4.2. If $0 \leq p \leq m$, the map $\widetilde{\Delta}_{p}$ in (4.4) induces a morphism

$$
\mathcal{V}_{\lambda}^{m} \rightarrow \mathcal{V}_{\lambda-2 p}^{m-p}
$$

of vector bundles in (4.6) over $X=\Gamma \backslash \mathcal{H}$.

Proof. Given $\lambda \in \mathbb{Z}$ and $p \in\{0,1, \ldots, m\}$, it suffices to prove that

$$
\widetilde{\Delta}_{p}\left(\gamma \odot_{\lambda}^{m}(z, f(X))\right)=\gamma \odot_{\lambda-2 p}^{m-p} \widetilde{\Delta}_{p}(z, f(X))
$$

for all $z \in \mathcal{H}, \gamma \in \Gamma$ and $f(X) \in \mathbb{C}_{m}[X]$. If $z \in \mathcal{H}, \gamma \in \Gamma$ and

$$
f(X)=\sum_{r=0}^{m} c_{r} X^{r} \in \mathbb{C}_{m}[X],
$$

using (3.6), (4.2) and (4.5), we obtain

$$
\begin{aligned}
& \widetilde{\Delta}_{p}\left(\gamma \odot_{\lambda}^{m}(z, f(X))\right) \\
& =\widetilde{\Delta}_{p}\left(\gamma z, \sum_{r=0}^{m} \sum_{k=r}^{m}\left(\begin{array}{l}
k \\
r
\end{array}\right) \mathfrak{J}(\gamma, z)^{\lambda-2 r} \mathfrak{K}(\gamma, z)^{k-r} c_{k} X^{r}\right) \\
& \quad=\left(\gamma z, \sum_{r=0}^{m-p}\left(\begin{array}{c}
r+p \\
p
\end{array}\right) \sum_{k=r+p}^{m}\left(\begin{array}{c}
k \\
r+p
\end{array}\right) \mathfrak{J}(\gamma, z)^{\lambda-2 r-2 p} \mathfrak{K}(\gamma, z)^{k-r-p} c_{k} X^{r}\right) \\
& =\left(\gamma z, \sum_{r=0}^{m-p} \sum_{k=r}^{m-p}\left(\begin{array}{c}
r+p \\
p
\end{array}\right)\left(\begin{array}{c}
k+p \\
r+p
\end{array}\right) \mathfrak{J}(\gamma, z)^{\lambda-2 r-2 p} \mathfrak{K}(\gamma, z)^{k-r} c_{k+p} X^{r}\right) .
\end{aligned}
$$

On the other hand,

$$
\begin{aligned}
\gamma & \odot_{\lambda-2 p}^{m-p} \widetilde{\Delta}_{p}(z, f(X)) \\
& =\gamma \odot_{\lambda-2 p}^{m-p}\left(z, \sum_{r=0}^{m-p}\left(\begin{array}{c}
r+p \\
p
\end{array}\right) c_{r+p} X^{r}\right) \\
& =\left(\gamma z, \sum_{r=0}^{m-p} \sum_{k=r}^{m-p}\left(\begin{array}{c}
k+p \\
p
\end{array}\right) \Xi_{r}^{\lambda-2 p}(\gamma, z) X^{r}\right) \\
& =\left(\gamma z, \sum_{r=0}^{m-p} \sum_{k=r}^{m-p}\left(\begin{array}{c}
k+p \\
p
\end{array}\right)\left(\begin{array}{c}
k \\
r
\end{array}\right) \mathfrak{J}(\gamma, z)^{\lambda-2 r-2 p} \mathfrak{K}(\gamma, z)^{k-r} c_{k+p} X^{r}\right) \\
& =\left(\gamma z, \sum_{r=0}^{m-p} \sum_{k=r}^{m-p}\left(\begin{array}{c}
r+p \\
p
\end{array}\right)\left(\begin{array}{c}
k+p \\
r+p
\end{array}\right) \mathfrak{J}(\gamma, z)^{\lambda-2 r-2 p} \mathfrak{K}(\gamma, z)^{k-r} c_{k+p} X^{r}\right) ;
\end{aligned}
$$

hence, the theorem follows. 
REMARK 4.3. If $p=m$ in (4.7), then we obtain the morphism

$$
\mathcal{V}_{\lambda}^{m} \rightarrow \mathcal{V}_{\lambda-2 m}^{0}
$$

from a vector bundle to a line bundle, where the holomorphic sections of the line bundle $\mathcal{V}_{\lambda-2 m}^{0}$ can be identified with modular forms as in Remark 3.4.

\section{References}

[1] Y. Choie and M. H. Lee, 'Quasimodular forms, Jacobi-like forms, and pseudodifferential operators', Preprint.

[2] A. Eskin and A. Okounkov, 'Asymptotics of numbers of branched coverings of a torus and volumes of moduli spaces of holomorphic differentials', Invent. Math. 145 (2001), 59-103.

[3] M. Kaneko and D. Zagier, A Generalized Jacobi Theta Function and Quasimodular Forms, Progress in Mathematics, 129 (Birkhäuser, Boston, 1995), pp. 165-172.

[4] A. Okounkov and R. Pandharipande, 'Gromov-Witten theory, Hurwitz theory, and completed cycles', Ann. of Math. 163 (2006), 517-560.

[5] E. Royer, 'Evaluating convolution sums of the divisor function via quasimodular forms', Int. J. Number Theory 21 (2007), 231-262.

MIN HO LEE, Department of Mathematics, University of Northern Iowa, Cedar Falls, IA 50614, USA

e-mail: lee@math.uni.edu 